\title{
The Vasorelaxant Properties of Novel Benzodiazepine-like Ligands on Isolated rat Thoracic Aorta
}

\author{
Milica Gajić Bojić, ${ }^{1}$ Miroslav Savić ${ }^{2}$
}

\section{Abstract}

Background/Aim: In addition to well-established central effects, benzodiazepines, but also some other allosteric modulators of gamma-amino-butyric acid (GABA) receptor exhibit significant vascular effects. However, there are currently no elucidated mechanisms for manifested vasodilatory properties and very little is known about GABA gamma-amino-butyric acid function and $\mathrm{GABA}_{\mathrm{A}}$ receptor expression within peripheral blood vessels.

Methods: In the present study, we demonstrated the vasorelaxant properties of diazepam, GABA and novel imidazobenzodiazepine amide ligands GL-II-73 and GLII-74, which are characterized as positive allosteric modulators of $\alpha 5$ - containing $\mathrm{GABA}_{\mathrm{A}}$ receptor. Using isometric organ bath system, we examined the vascular responses to phenylephrine, in the presence and absence of various ligands, in the rat thoracic aorta.

Results: The observed significant and strong attenuation of the maximal contractile response of phenylephrine indicates a non-competitive antagonism of diazepam, GL-II-73 and GL-II-74 ( $\mathrm{p}<0.001)$, whereas GABA does not affect phenylephrine contraction.

Since the strongest inhibitory effect was observed with compound GL-II-74, that, compared to other tested ligands, exhibited a higher potentiation at $\alpha 5 \mathrm{GABA}_{\mathrm{A}} \mathrm{Rs}$, it could be assumed that the $\alpha 5$ subunit plays a significant role in the structure of putatively present "vascular" GABA Rs.

Conclusion: This work emphasizes the importance of $\mathrm{GABA}_{\mathrm{A}} \mathrm{Rs}$ research in the periphery and also points to the possibility of using $\alpha 5$ selective $G_{A B A_{A}}$ R modulators as potential therapeutic targets for novel vasodilators.

Key words: GABA A receptor, positive allosteric modulators, vasodilatation, rat thoracic aorta.
(1) Center for biomedical research, Faculty of Medicine, University of Banja Luka, Banja Luka, the Republic of Srpska, Bosnia and Herzegovina.

(2) Department of Pharmacology, Faculty of Pharmacy, University of Belgrade, Belgrade, Serbia.
Correspondence:

MILICA GAJIĆ BOJIĆ

E: milica.gajic@med.unibl.org

\section{ARTICLE INFO}

Received: 3 June 2020 Revision received: 12 June 2020 Accepted: 15 June 2020

\section{Introduction}

In addition to being a major inhibitory neurotransmitter in the central nervous system (CNS), GABA has a functional importance in many peripheral tissues. Peripheral GABA regulation of cardiovascular function has long been known, ${ }^{1,2}$ but to date no distinct roles or exact mechanisms have been established.
The first studies with isolated cerebral blood vessels had suggested that $\mathrm{GABA}_{A}$ receptors $\left(\mathrm{GABA}_{\mathrm{A}} \mathrm{Rs}\right)$ exist in vascular smooth muscle, where GABA or GABA-agonists produced a dilatation of cerebral arteries. ${ }^{3,4}$ Even though $\mathrm{GABA}_{\mathrm{A}} \mathrm{R}$ subunit mRNA expression has been demonstrated in various rat peripheral organs, such as kidneys, adrenal gland, ovary, testis, uterus and ileum ${ }^{5,6}$ very lit- 
tle is known about $\mathrm{GABA}_{\mathrm{A}} \mathrm{R}$ expression and GABA function within the peripheral vascular smooth muscle.

GABA levels in the peripheral vessels and activity of GABA-related enzymes, especially glutamic acid decarboxylase (GAD) and gamma-aminobutyric acid-transaminase (GABA-T), have been found to be up to $1 \%$ of those in the brain ${ }^{7}$ and such a modest expression can be regarded as insufficient to directly elicit vasoactivity of GABA. However, the finding that cultured human aortic and umbilical vein endothelial cells synthesize GABA, which further exhibits direct effects on endothelial cell metabolism ${ }^{8}$ indicates the potential role of GABA as an autocoid for neighbouring smooth muscle cells.

Benzodiazepines (BZs) as positive modulators of $\mathrm{GABA}_{A}$ Rs have a wide range of acute effects, such as anxiolytic, sedative, hypnotic, skeletal muscle relaxant, anticonvulsant, anterograde amnesic and ataxic action. In addition to well-established central role, BZ's also exhibit vasodilatory properties. ${ }^{9,10,11}$ However, there are currently no elucidated mechanisms of BZ`s vasoactivity and propensity to reduce the intracellular influx of calcium into the smooth muscle cell.

Vascular effects similar to those of diazepam are also exhibited by other $\mathrm{GABA}_{\mathrm{A}} \mathrm{R}$ allosteric modulators, such as endogenous neurosteroids. ${ }^{12}$ Considering that the peripheral benzodiazepine receptor (officially known as translocator protein, TSPO) has no role in regulating smooth muscle contractility, ${ }^{10}$ the published results suggest that activation or positive modulation of $\mathrm{GABA}_{\mathrm{A}} \mathrm{Rs}$, such as that effected by diazepam, result in vascular dilation..$^{12}$ However, the receptor subtype substrate of that action is totally unknown.

Herein, the vasorelaxant properties of novel ligands with imidazobenzodiazepine (IBZD) amide structure GL-II-73 and GL-II-74 were demonstrated, which are characterised as positive allosteric modulators (PAMs) of $\mathrm{GABA}_{A} \mathrm{R}$ with preferential potentiation at $\alpha 5$ subunit-containing receptors. ${ }^{13}$ In order to examine their possible vasoactivity, isometric organ bath study of vascular responses to phenylephrine was conducted. Diazepam and GABA were used in the same protocols, and in this way the manifested effects were compared and thus the possible mechanisms of vasoactivity were assessed.

\section{Methods}

\section{Vessel preparation}

Wistar rats were obtained from the Military Medical Academy and housed in vivarium facilities of the Faculty of Pharmacy, University of Belgrade (Belgrade, Serbia) under normal housing conditions (temperature: $22 \pm 1{ }^{\circ} \mathrm{C}$, relative humidity: 40-70\%,12/12 h light/dark period). As a part of a wider national project led by the senior author, the experiments were approved by the Ethical Council for the Protection of Experimental Animals of the Ministry of Agriculture, Forestry and Water Management of the Republic of Serbia. Male rats were anaesthetised with combination of ketamine hydrochloride $(90 \mathrm{mg} /$ kg, Ketamidor, Richter Pharma AG, Wels, Austria) and xylazine hydrochloride $(10 \mathrm{mg} / \mathrm{kg}$, Xylased, Bioveta, A. S., Ivanovice na Hane, Czech Republic). The descending thoracic aortas were dissected and cleared of surrounding adipose and connective tissue.

Aortic rings of approximately $3 \mathrm{~mm}$ length were obtained from isolated blood vessels bathed in Petri dish containing chilled $\left(4^{\circ} \mathrm{C}\right)$ modified Krebs-bicarbonate solution (composition: 118.3 $\mathrm{mM} \mathrm{NaCl}, 4.7 \mathrm{mM} \mathrm{KCl}, 2.5 \mathrm{mM} \mathrm{CaCl}, 1.2 \mathrm{mM}$ $\mathrm{MgSO}_{4}, 25 \mathrm{mM} \mathrm{NaHCO}_{3}, 1.2 \mathrm{mM} \mathrm{KH}_{2} \mathrm{PO}_{4}, 11 \mathrm{mM}$ glucose). ${ }^{14}$ The aortic rings were rapidly placed for measurement of isometric contraction.

\section{Experiments with isolated vascular rings}

The aortic rings were suspended between two wire hooks in organ bath chambers filled with 15 $\mathrm{mL}$ modified Krebs-bicarbonate solution $\left(37^{\circ} \mathrm{C}\right.$, $\mathrm{pH} 7.40$ ) aerated with mixture of $95 \%$ oxygen $/ 5$ $\%$ carbon dioxide. The upper hook was connected to the MLT0201 force displacement transducer (Panlab, Spain), and changes in isometric force were recorded using PowerLab/4SP data acquisition system (AD Instruments, Castle Hill, Australia) and software LabChart 7 Pro (AD Instruments). Experiments were performed on four organ baths in parallel.

The rings were placed under the optimal passive stretching tension of $4.0 \mathrm{~g}$, defined previously. ${ }^{15}$ The equilibration period of the preparation lasted $60 \mathrm{~min}$ and during that time the bathing solution was changed every $10 \mathrm{~min}$. Each aortic ring was subjected first to the initial challenging contraction with potassium chloride $\left(6 \times 10^{-2} \mathrm{M}\right)$ to assess the viability of preparations. The rings 
was then left to re-equilibrate for $40-50 \mathrm{~min}$, before the appropriate protocol procedures were used.

Experimental protocol: experiments were aimed to investigate the effects of diazepam, GABA and novel imidazobenzodiazepine (IBZD) amide ligands (GL-II-73 and GL-II-74) on the contractile response induced by the $\alpha 1$ adrenoreceptor agonist phenylephrine (PE), in the endothelium-intact aortic rings.

At the beginning of the protocol, to obtain a reference contraction, the contractile response induced by potassium $\left(6 \times 10^{-2} \mathrm{M}\right)$ was measured. After preparations were washed-out several times until tone returned to baseline, concentration-response curve of PE (control curve) was generated $\left(10^{-9}-10^{-4} \mathrm{M}\right)$. Aortic ring had been washed-out again and test compound (each at concentration $10^{-4} \mathrm{M}$ and $10^{-5} \mathrm{M}$, except for diazepam with the applied concentration of $10^{-5} \mathrm{M}$ ) were added individually to the organ bath, 60 min before another PE-induced contraction was obtained. The effects of the test compound on the PE contraction were assessed by comparing the contractile response in the presence or absence of compound. Results were expressed in relation to the contraction achieved by the same ring previously contracted with isotonic potassium.

\section{Drugs and solutions}

Phenylephrine hydrochloride and GABA were purchased from Sigma-Aldrich (St. Louis, USA). Diazepam was generously supplied by Galenika (Belgrade, Serbia).

The ligands GL-II-73 ((R)-8-Ethynyl-6-(2-fluorophenyl)-N,N,4-trimethyl-4H-benzo[f]imidazo[1,5- $a][1,4]$ diazepine-3-carboxamide) and GL-II-74 ((R)-N-Ethyl-8-ethynyl-6-(2-fluorophenyl)-4-methyl-4H-benzo[ $f$ ]imidazo[1,5-a] $[1,4]$ diazepine-3-carboxamide) were synthesised at the Department of Chemistry and Biochemistry, University of Wisconsin, Milwaukee, USA.

All drugs were prepared as concentrated stock solutions $10^{-1} \mathrm{M}$ in $100 \%$ ethyl alcohol, with exception for PE and GABA, the stocks of which were prepared in distilled water. The subsequent dilutions were carried out in mixture of solvent and distilled water, so that the final solvent concentration was never higher than $0.3 \%$ in the 15 mL-organ bath.

\section{Statistical analysis}

Statistical analysis and graphs were prepared using LabChart 7 Pro software (AD Instruments) and SigmaPlot 11 (Systat Software Inc.) Results were summarised as the mean \pm standard error of $n$ replicates, where $n$ is the number of aortic rings tested in one protocol, each obtained from a separate animal. The negative logarithm of the ligand concentration $\left(\mathrm{pEC}_{50}\right)$ producing $50 \%$ of the maximum response was calculated in LabChart 7 Pro software. Statistical analyses were performed using Student's paired t-test ( $p$ values less than 0.05 were considered statistically significant).

\section{Results}

Diazepam $\left(10^{-5} \mathrm{M}\right)$ produced a significant attenuation $(p<0.001)$ of the maximal contractile re-

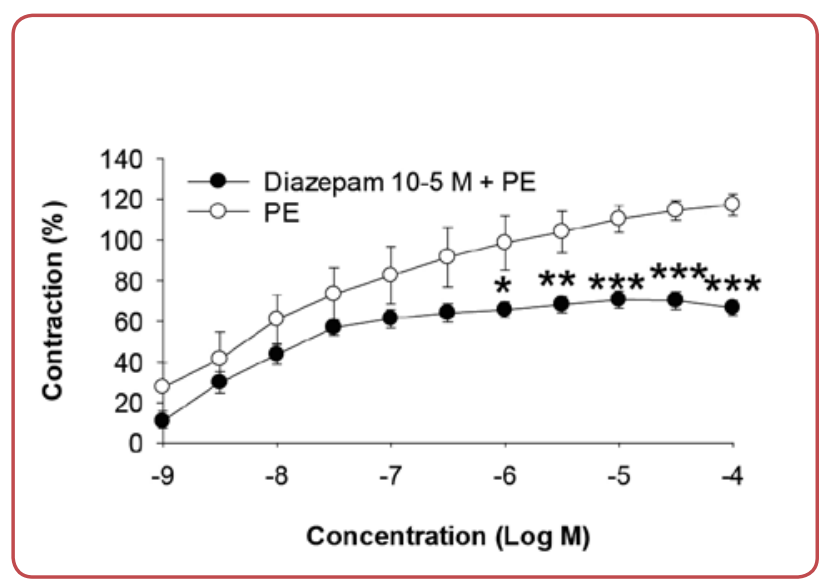

Figure 1. Effect of diazepam on the phenylephrine concentration-response curve when aortic rings were pre-incubated with diazepam $\left(10^{-5} M, n=6\right)$. Results (mean \pm SEM) are expressed with reference to the contraction induced by potassium $\left(6 \times 10^{-2} \mathrm{M}\right)$. ${ }^{* \star *} p<0.001$; ${ }^{* *} p<0.01$; ${ }^{*} p<0.05$ (significantly different $E_{\max }$ values). Parentheses indicate the number of preparations studied, each obtained from a separate animal.

sponse of PE $(117.24 \pm 5.30 \%$ vs $66.62 \pm 3.71 \%)$, while it did not affect the $\mathrm{pEC}_{50}$ value of PE (Figure 1).

Although applied at a very high concentration $\left(10^{-4}\right.$ M), GABA did not shift the PE concentration-response curve or affect the PE-induced maximal contraction (Figure 2A). GABA used at concentration of $10^{-5} \mathrm{M}$ also did not affect the PE contraction (data not shown).

The ligand GL-II-73 $\left(10^{-5} \mathrm{M}\right)$ significantly decreased $(p<0.05)$ the maximal contractile re- 


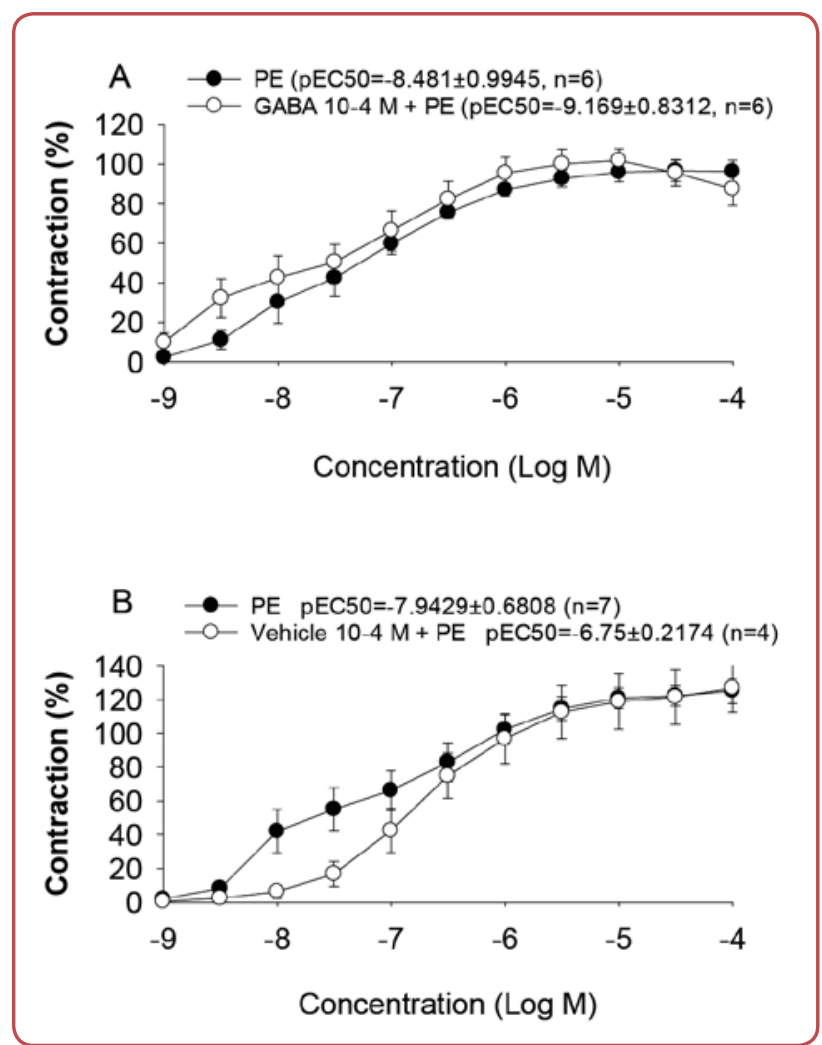

Figure 2. Cumulative log concentration-relaxation curves for pheny-lephrine (PE) in the absence and presence of $A$ ) GABA $10^{-4}$ $M(n=6)$ vehicle $10^{-4} M(n=4)$. Results (mean \pm SEM) are expressed with reference to the contraction induced by potassium $\left(6 \times 10^{-2} \mathrm{M}\right)$. Parentheses indicate the number of preparations studied, each obtained from a separate animal.

sponse to PE $(82.36 \pm 16.41 \%$ vs $125.17 \pm 7.45$ $\%)$, but had no effect on the PE potency (there was no significant differences between $\mathrm{pEC}_{50}$ values). Pre-treatment with GL-II-73 at a ten-fold higher concentration $\left(10^{-4} \mathrm{M}\right)$ strongly attenuated $(\mathrm{p}<$ $0.001)$ the maximal PE contraction $(35.03 \pm 9.60$ $\%$ vs $125.17 \pm 7.45 \%$ ), whereas had no significant effect on the $\mathrm{pEC}_{50}$ value of PE $\left(10^{-9}-10^{-4} \mathrm{M}\right)$ (Figure $3 \mathrm{~A}, \mathrm{~B})$.

The ligand GL-II-74 used at the higher concentration $\left(10^{-4} \mathrm{M}\right)$ strongly decreased $(\mathrm{p}<0.001)$ the PE-induced maximal contraction, compared with untreated rings $(18.08 \pm 4.48 \%$ vs 125.17 $\pm 7.45 \%$ ). When aortic rings were pre-treated with a lower concentration of GL-II-74 $\left(10^{-5} \mathrm{M}\right)$ there also was no effect on the $\mathrm{pEC}_{50}$ value for PE, while the maximal contractile response was significantly $(\mathrm{p}<0.001)$ decreased $(42.99 \pm 11.63 \%$ vs $125.17 \pm 7.45 \%$ ) (Figure $3 \mathrm{C}$, D).

The influence of vehicle was obtained when the same volume, as for ligand additions at concentration $\left(10^{-4} \mathrm{M}\right)$, was added in pre-incubation period. There was no altered pharmacological activity on aortic rings in presence of vehicle (Figure 2B).

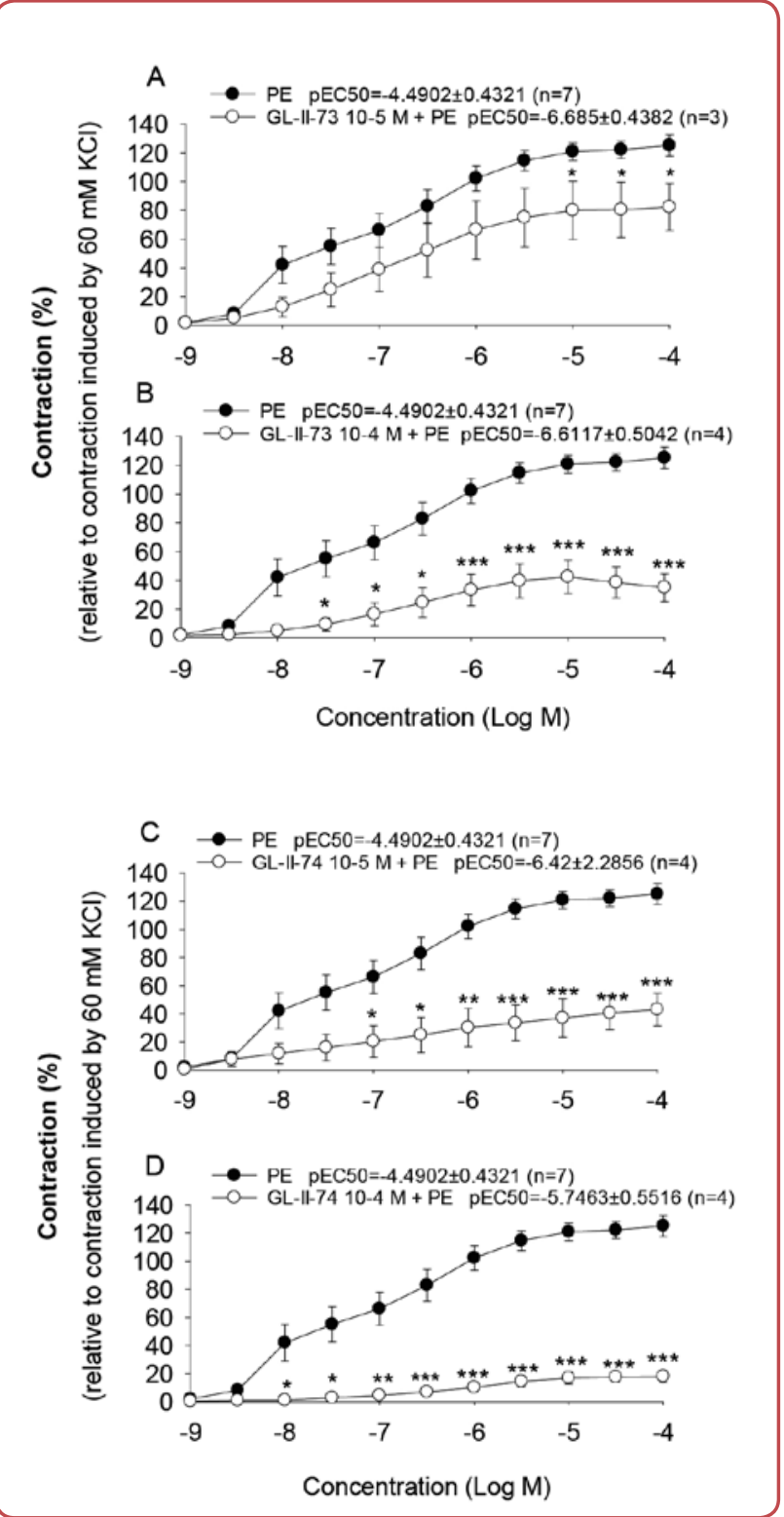

Figure 3. Cumulative log concentration-relaxation curves for phenylephrine (PE) in the absence and presence of $A)$ GL-II-73 10-5 $M(n=$ 3); B) GL-II-73 10-4 M ( $n=4)$; C) GL-II-74 10-5 M (n=4); D) GL-II-74 10-4 $M(n=4)$. Results (mean $\pm S E M)$ are expressed with reference to the contraction induced by potassium $\left(6 \times 10^{-2} \mathrm{M}\right)$.

${ }^{* * *} p<0.001$; ${ }^{* \star} p<0.01 ;{ }^{*} p<0.05$ (significantly different $E_{\max }$ values). Parentheses indicate the number of preparations studied, each obtained from a separate animal.

\section{Discussion}

The differential expression of total of nineteen GABA $_{A}$ R subunits ( $\left.\alpha 1-6, \beta 1-3, \gamma 1-3, \delta, \varepsilon, \theta, \pi, \rho 1-3\right)$ has been demonstrated in various peripheral organs, indicating that $\mathrm{GABA}_{\mathrm{A}} \mathrm{R}$ subunits are expressed in a tissue-specific manner. ${ }^{6,16}$ Immunohistochemical analyses, western blotting and real time reverse transcription polymerase chain 
reaction (RT-PCR), had revealed the presence of functional $\mathrm{GABA}_{\mathrm{A}}$ Rs within the gastrointestinal tract, ${ }^{16}$ airway smooth muscle of trachea, ${ }^{17,18}$ pancreatic $\beta$ cells. ${ }^{19}$ However, there is still no clear evidence for the expression of functional $\mathrm{GABA}_{A} \mathrm{Rs}$ on vascular smooth muscle cells. This study was based on the hypothesis that positive allosteric modulation of GABA ${ }_{A}$ Rs that contain the $\alpha 5$ subunit contributes to vasodilating effects of BZs. The inhibiting influence of diazepam on the contractile activity of phenylephrine in isolated rat aorta was demonstrated, thus confirming the previous in vitro studies, where diazepam inhibited PE- induced calcium oscillations, ${ }^{20}$ attenuated the PE- induced contractions in the rat aorta ${ }^{10}$ and produced vasodilation in the PE-precontracted rat aortic rings. ${ }^{9}$ The observed significant and strong attenuation of the maximal contractile response of PE indicates a non-competitive antagonism of diazepam, in terms of signalling mechanisms of contraction in vascular smooth muscle cells.

Concentration of GABA in the systemic circulation of humans was found to be between 0.5 to $3 \mu \mathrm{M}$. ${ }^{8}$ It has been suggested that apart from GABA produced by the pancreatic beta cells, adrenal gland and certain immune cells, an important source of GABA in circulation may be that related to endothelial cells of blood vessels. ${ }^{8}$ The examination of the effect of GABA on vascular response to PE in isolated rat aorta indicated that GABA did not affect $P E$ contraction, even when applied in high concentration $(100 \mu \mathrm{M})$. Findings of GABA indifference on contracted aortic rings found in this study may correlate with earlier data that no vasodilating effects on peripheral blood vessels have been reported for GABA. ${ }^{8,11,21}$ Nevertheless, the results from other studies with isolated blood vessels have shown that GABA has relaxatory effect on rat mesenteric bed. ${ }^{22,23}$

Diazepam, a standard non-selective PAM of $\mathrm{GABA}_{\mathrm{A}} \mathrm{Rs}$, was used as the reference ligand, in order to investigate the vasorelaxant properties of GL-II-73 and GL-II-74. Previously performed electrophysiological and binding studies showed that ligands GL-II-73 and GL-II-74 acted as PAMs with primary efficacy and affinity at $\alpha 5$-containing $\mathrm{GABA}_{\mathrm{A}} \mathrm{Rs}^{13}{ }^{13}$ whereas diazepam modulates GAB$A_{A} R$ activity as a non-selective PAM, with high affinity and efficacy at $\alpha 1, \alpha 2, \alpha 3$ or $\alpha 5$-containing $\mathrm{GABA}_{\mathrm{A}} \mathrm{Rs}^{.24}$ Both ligands (GL-II-73 and GL-II-74) reduced the maximum contraction induced by $\mathrm{PE}$, compared to the untreated rings, indicating similarity to the effects of diazepam in the same protocol.

It was also shown that the vascular responses to $\mathrm{PE}$ in the isolated aortic rings vary significantly, depending on concentrations of GL-II-73 and GLII-74 used during incubation. When aortic rings were pre-treated with a higher concentration $\left(10^{-4} \mathrm{M}\right)$, the maximal contractile response was approximately $20-30 \%$ of the corresponding control maximal contraction ie (without the presence of ligand), while at lower concentrations of tested ligand, the inhibitory effects were weaker (approximately $50 \%$ reduction in contraction). This clearly indicates a concentration-dependent inhibitory effects of the tested IBZDs.

Concentrations of compounds used in this study were in accordance with those in studies of vascular effects of BZs on isolated blood vessels. Although these concentrations are too high to correspond with the clinical use of BZs, they can still be reached in cases of overdose or other abuse. ${ }^{11}$ In this regard, their vascular effects should not be neglected. Interestingly, a stronger inhibitory effect on the PE concentration-response curve was observed with compound GL-II-74 than with GL-II-73. This might be explained by the observed differences in their modulatory properties, taking into account that GL-II-74 exhibited a higher potentiation at $\alpha 5 \mathrm{GABA}_{\mathrm{A}}$ Rs than GL-II-73. ${ }^{13} \mathrm{Ac}$ cordingly, it could be assumed that the presence of the $\alpha 5$ subunit in the structure of putatively present "vascular" GABA ${ }_{A}$ Rs may play a substantial role in the overall observed vasoactivity.

\section{Conclusion}

The present work highlights the importance of $\mathrm{GABA}_{\mathrm{A}} \mathrm{Rs}$ research in the periphery and also opens the possibility of using $\alpha 5$ selective $\mathrm{GABA}_{\mathrm{A}} \mathrm{R}$ modulators as potential therapeutic targets for novel vasodilators.

\section{Acknowledgements}

This work was supported in part by the Ministry of Education, Science and Technological Development, the Republic of Serbia - Grant No. 175076 (MMS). 


\section{Conflict of interest}

None.

\section{References}

1. Billingley M, Suria A, Gilman R, Shokes L, Shahvari M. Evidence for GABA involvement in the peripheral control of blood pressure and vascular resistance. Brain Res Bull 1980;5 Suppl 2:329-33.

2. Lim DY, Suh J, Yoo HJ, Kim WS, Kim SB, Lee SK, et al. Influence of gamma-aminobutyric acid on the changes of blood pressure in rats. Korean J Intern Med 1990 Jan;5(1):23-33.

3. Fujiwara M, Muramatsu I, Shibata S. $\gamma$-Aminobutyric acid receptor on vascular smooth muscle of dog cerebral arteries. Br J Pharmacol 1975 Dec;55(4):561-2.

4. Edvinsson L, Krause DN. Pharmacological characterization of GABA receptors mediating vasodilation of cerebral arteries in vitro. Brain Res 1979 Sep 7;173(1):8997.

5. Akinci MK, Schofield PR. Widespread expression of GAB$\mathrm{A}(\mathrm{A})$ receptor subunits in peripheral tissues. Neurosci Res 1999 Nov; 35(2):145-53.

6. Takano K, Yatabe MS, Abe A, Suzuki Y, Sanada H, Watanabe $\mathrm{T}$, et al. Characteristic expressions of GABA receptors and GABA producing/transporting molecules in rat kidney. PLoS One 2014 Sep 4;9(9):e105835. doi: 10.1371/journal.pone.0105835.

7. Hamel E, Krause DN, Roberts E. Specific cerebrovascular localization of glutamate decarboxylase activity. Brain Res 1981 Oct 26;223(1):199-204.

8. Sen S, Roy S, Bandyopadhyay G, Scott B, Xiao D, Ramadoss $S$, et al. $\gamma$-Aminobutyric acid Is synthesized and released by the endothelium: potential implications. Circ Res 2016 Aug 19;119(5):621-34.

9. Galindo A, Vargas ML, Estan JG, Fuentes T, Hernandez J. Synergistic interaction of diazepam with 3 ',5'-cyclic adenosine monophosphate-elevating agents on rat aortic rings. Eur J Pharmacol 2001 Oct 5;428(2):269-75.

10. Park SE, Sohn JT, Kim C, Chang KC, Shin IW, Park KE, et al. Diazepam attenuates phenylephrine-induced contractions in rat aorta. Anesth Analg 2006 Mar;102(3):682-9.

11. Colussi GL, Fabio AD, Catena C, Chiuch A, Sechi LA. Involvement of endothelium-dependent and -independent mechanisms in midazolam-induced vasodilation. Hypertens Res 2011 Aug;34(8):929-34.
12. Jacob MK, White RE. Diazepam, gamma-aminobutyric acid, and progesterone open $\mathrm{K}(+)$ channels in myocytes from coronary arteries. Eur J Pharmacol $2000 \mathrm{Sep}$ 8;403(3):209-19.

13. Prevot TD, Li G, Vidojevic A, Misquitta KA, Fee C, Santrac $A$, et al. Novel benzodiazepine-like ligands with various anxiolytic, antidepressant, or pro-cognitive profiles. Mol Neuropsychiatry 2019 Apr;5(2):84-97.

14. Rizvić E, Janković G, Savić M. Elucidation of the profound antagonism of contractile action of phenylephrine in rat aorta effected by an atypical sympathomimetic decongestant. Korean J Physiol Pharmacol 2017;21(4):385-95.

15. Jespersen B, Tykocki NR, Watts SW, Cobbett PJ. Measurement of smooth muscle function in the isolated tissue bath-applications to pharmacology research. J Vis Exp 2015 Jan 19;(95):52324. doi: 10.3791/52324.

16. Seifi M, Brown JF, Mills J, Bhandari P, Belelli D, Lambert JJ, et al. Molecular and functional diversity of GABA-A receptors in the enteric nervous system of the mouse colon. J Neurosci 2014 Jul 30;34(31):10361-78.

17. Mizuta K, Xu D, Pan Y, Comas G, Sonett JR, Zhang Y, et al. GABAA receptors are expressed and facilitate relaxation in airway smooth muscle. Am J Physiol Lung Cell Mol Physiol. 2008 Jun;294(6):L1206-16. doi:10.1152/ ajplung.00287.2007.

18. Forkuo GS, Guthrie ML, Yuan NY, Nieman AN, Kodali $\mathrm{R}$, Jahan R, et al. Development of GABAA receptor subtype-selective imidazobenzodiazepines as novel asthma treatments. Mol Pharm 2016 Jun 6;13(6):2026-52.

19. Korol SV, Jin Z, Jin Y, Bhandage AK, Tengholm A, Gandasi NR, et al. Functional characterization of native, high-affinity GABA(A) receptors in human pancreatic $\beta$ Cells. EBioMedicine 2018 Apr;30:273-282.

20. Hong SJ, Damron DS, Murray PA. Benzodiazepines differentially inhibit phenylephrine-induced calcium oscillations in pulmonary artery smooth muscle cells. Anesthesiology 1998 Mar;88(3):792-9.

21. Perusquía M, Hernández R, Morales MA, Campos MG, Villalón CM. Role of endothelium in the vasodilating effect of progestins and androgens on the rat thoracic aorta. Gen Pharmacol 1996 Jan;27(1):181-5.

22. Farsi L, Keshavarz M, Soltani N. Relaxatory effect of gamma-aminobutyric acid (GABA) is mediated by same pathway in diabetic and normal rat mesenteric bed vessel. Iran J Bas Med Sci 2011;14(1):94-8.

23. Kharazmi F, Soltani N, Rezaei S, Keshavarz M, Farsi L. Role of GABAB receptor and L-Arg in GABA-induced vasorelaxation in non-diabetic and streptozotocin-induced diabetic rat vessels. Iran Biomed J 2015;19(2):91-5.

24. Sigel E, Ernst M. The benzodiazepine binding sites of GABA(A) receptors. Trends Pharmacol Sci 2018 Jul;39(7):659-71. 